\title{
Desenvolvimento de um programa para registro e avaliação de incidentes em radioterapia por feixe externo de fótons
}

\section{Development of a software for recording and evaluating incidents in external photon beam radiotherapy}

DOI: $10.54022 /$ shsv3n1-022

Recebimento dos originais: 01/01/2021

Aceitação para publicação: 31/01/2022

\section{André Luis de Carvalho Ribeiro}

Divisão de Física Médica, Instituto de Radioproteção e Dosimetria - IRD/CNEN E-mail: andhreluiz@gmail.com

Flavia Cristina da Silva Teixeira

Divisão de Aplicações Médicas e Pesquisa, Comissão Nacional de Energia

Nuclear - CNEN

E-mail: flavia@cnen.gov.br

\section{Eduardo de Paiva}

Divisão de Física Médica, Instituto de Radioproteção e Dosimetria - IRD/CNEN E-mail: edup2112@gmail.com

\section{RESUMO}

A radioterapia com o uso de feixes de fótons produzidos por aceleradores lineares experimentou importantes avanços tecnológicos nas últimas décadas, contribuindo para melhorar a qualidade do tratamento de pacientes com câncer. Neste sentido, a aprendizagem com incidentes ganhou grande destaque durante sucessivas abordagens sobre a segurança do paciente, resultando na criação de ferramentas voltadas para essa finalidade. No Brasil, ainda existem poucos estudos realizados sobre essa abordagem na radioterapia $e$, diante do crescimento da oncologia no país e do aumento constante das estimativas de novos casos, foi observada a necessidade da criação de um sistema de registro e aprendizagem com incidentes a fim de promover melhorias no atendimento, eficácia no tratamento e proporcionar aprimoramento dos profissionais. Neste trabalho apresentamos o desenvolvimento de uma ferramenta para registro e análise de incidentes que seja voltada para a realidade do país. Foram realizadas etapas que envolvem a análise dos mapas de processo no tratamento para 0 enriquecimento do sistema (chamado de Sistema Padronizado de Incidentes em Radioterapia - SPIRad). Durante a aplicação, foi possível observar que as instituições selecionadas possuem alguns fatores em comum que foram úteis para a análise. Após a conclusão do desenvolvimento do SPIRad e sua aplicação foi possível afirmar que o sistema poderá ser de grande utilidade no aprimoramento de protocolos de tratamento, assim como auxiliará na política de gestão de qualidade e na cultura de segurança do paciente.

Palavras-chave: Radioterapia, proteção radiológica, incidentes, proteção do 
paciente, gestão de qualidade.

\section{ABSTRACT}

Radiotherapy using photon beams produced by linear accelerators has experienced important technological advances in recent decades, contributing to improve the quality of treatment for cancer patients. In this sense, learning from incidents has gained great prominence during successive approaches to patient safety, resulting in the creation of tools aimed at this purpose. In Brazil, there are still few studies carried out on this approach in radiotherapy and, given the growth of oncology in the country and the constant increase in the estimates of new cases, the need to create a recording and learning system with incidents was observed in order to promote improvements in care, effectiveness in treatment and provide professional improvement. In this work we present the development of a tool for recording and analyzing incidents that is focused on the reality of the country. Steps were carried out involving the analysis of process maps in the treatment to enrich the system (called the Standardized System of Incidents in Radiotherapy SPIRad). During the application, it was possible to observe that the selected institutions have some factors in common that were useful for the analysis. After completing the development of SPIRad and its application, it was possible to affirm that the system could be of great use in improving treatment protocols, as well as helping in the quality management policy and in the patient safety culture.

Keywords: Radiotherapy, radiation protection, incidents, patient protection, quality management.

\section{INTRODUÇÃO}

A radioterapia é uma técnica que utiliza radiação ionizante para 0 tratamento de neoplasias e adquire grande importância quando se leva em conta que pelo menos $52 \%$ dos pacientes oncológicos realizam o tratamento via radioterapia em algum estágio do seu tratamento [BEGG et al., 2011]. Contudo, mesmo com os avanços tecnológicos na radioterapia com o uso de feixes provenientes de aceleradores lineares, muito ainda precisa ser feito para garantir o sucesso no tratamento. Em destaque estão os aspectos relativos à segurança devido à característica multidisciplinar das várias etapas do processo de tratamento radioterápico e da complexidade dos equipamentos envolvidos [DELANEY et al., 2005].

Desde a década de 90, foi desenvolvida uma maior consciência dos riscos no tratamento em radioterapia, especialmente os riscos que podem ser minimizados ou evitados [ICRP, 2000; HOLMBERG, 2006; HENDEE, 2011]. Então, ao longo dos últimos anos, foram tomadas iniciativas com o intuito de 
melhorar a segurança do paciente através de várias sessões e conferências em reuniões dedicadas a este tema [DONALDSON, 2007; FORD et al., 2012]. Após muitas abordagens para debater melhorias na segurança do paciente e na qualidade do tratamento, foi reconhecido que a aprendizagem com os incidentes tem um papel fundamental neste tema, dando início a um estudo em junho de 2010 pela American Association of Physicists in Medicine para promover 0 desenvolvimento e a utilização de sistemas de aprendizagem com incidentes em radioterapia [FORD et al., 2012]. A aprendizagem com incidentes refere-se a todo ciclo de feedbacks de relatos e, em seguida, análise e desenvolvimento de intervenções para evitar que o mesmo volte a acontecer.

Atualmente, a legislação nacional vigente que estabelece requisitos de segurança e proteção radiológica em radioterapia são descritos pela norma NN6.10 da CNEN [CNEN, 2014]. A seção VI, art. 26, determina que todo serviço de radioterapia deve estabelecer ações de resposta a incidentes (ou eventos adversos), de modo que "o titular, o responsável técnico e o especialista em física médica de radioterapia devem investigar imediatamente qualquer tratamento ou fração de tratamento terapêutico administrado a um paciente que seja diferente do planejado". Entretanto, no Brasil existem poucos estudos realizados sobre esse tipo de abordagem na radioterapia e, diante do crescimento da oncologia no país, do aumento constante no número de casos de câncer nas estimativas de biênio após biênio e do tratamento dos tumores sólidos na radioterapia, foi observada a necessidade da criação de um sistema de registro e aprendizagem sobre incidentes, a fim de promover melhorias no atendimento, eficácia no tratamento e proporcionar também o aprimoramento dos profissionais, o que já contribuiria diretamente para a diminuição dos incidentes. Portanto, entende-se que a formulação de um sistema de coleta de dados, que se encaixe na realidade e características dos serviços de radioterapia do país, poderá apontar as principais questões que, muitas vezes passam despercebidas e, somadas a fatores diversos, podem causar incidentes.

Para o desenvolvimento deste trabalho, foram escolhidas duas instituições no estado do Rio de Janeiro que oferecem serviços de radioterapia de alta complexidade devido à ampla variedade de técnicas de tratamento, ao número de equipamentos de terapia e à existência de uma equipe multidisciplinar. Além da 
exigência legal para se estabelecer uma metodologia sistemática de investigação de eventos adversos que atingem os pacientes, os serviços estavam se preparando para abordar essa prática, uma vez que o tema tem sido questionado frequentemente em palestras e workshops específicos para a área com a participação de representantes de instituições espalhadas pelo Brasil. Neste trabalho foi desenvolvida uma ferramenta de registro e avaliação de incidentes em radioterapia que seja voltada para a realidade do país.

\section{MATERIAIS E MÉTODOS}

A ferramenta foi desenvolvida em formato digital para que através de uma rede privada que assenta sobre a suíte de protocolos da internet (intranet), as clínicas que a utilizarem possam compartilhar informações importantes sobre eventos ocorridos entre elas. A ferramenta foi batizada de "Sistema Padronizado de Incidentes em Radioterapia (SPIRad)". O sistema foi dividido em 3 formulários sequenciais, Resumo do incidente (Figura 1), Análise do incidente (Figura 2 a 5), Formulário de resposta (Figura 6 e 7).

Figura 1: Formulário equivalente ao resumo de incidentes do SPIRad.

\begin{tabular}{|c|c|}
\hline \multicolumn{2}{|c|}{ 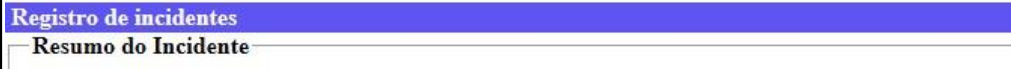 } \\
\hline 1. & Data do Incidente: $\mathrm{mm} / \mathrm{dd} / \mathrm{aaaa}$ \\
\hline 2. & Hora do Incidente: $-\cdots:--$ \\
\hline 3. & Data do Relatório: $\mathrm{mm} / \mathrm{dd} / \mathrm{aaaa}$ \\
\hline 4. & Data da Descoberta: $\mathrm{mm} / \mathrm{dd} / \mathrm{aaaa}$ \\
\hline 5 . & Tipo de Incidente: $\odot$ Incidente real $\bigcirc$ "Near-miss" \\
\hline 6. & Pessoa afetada: $\square$ Ninguém afetado $\square$ Um paciente afetado $\square$ Vários pacientes afetados \\
\hline 7. & Número de frações entregues incorretamente: [ \\
\hline 8. & Nome completo do paciente: $\square$ \\
\hline 9. & Número do registro médico do paciente: $\square$ \\
\hline 10. & Modalidade de tratamento: $\square$ Teleterapia $\square$ Braquiterapia \\
\hline & Nome completo da pessoa que reporta: \\
\hline & Papel da pessoa que reporta: \\
\hline & $\square$ Radio-oncologista $\square$ Físico Médico $\square$ Enfermeiro(a) $\square$ Técnico Radioterapia $\square$ Outros \\
\hline
\end{tabular}


Figura 2: Formulário equivalente a análise de incidentes do SPIRad com os campos específicos ao tipo de tratamento utilizado.

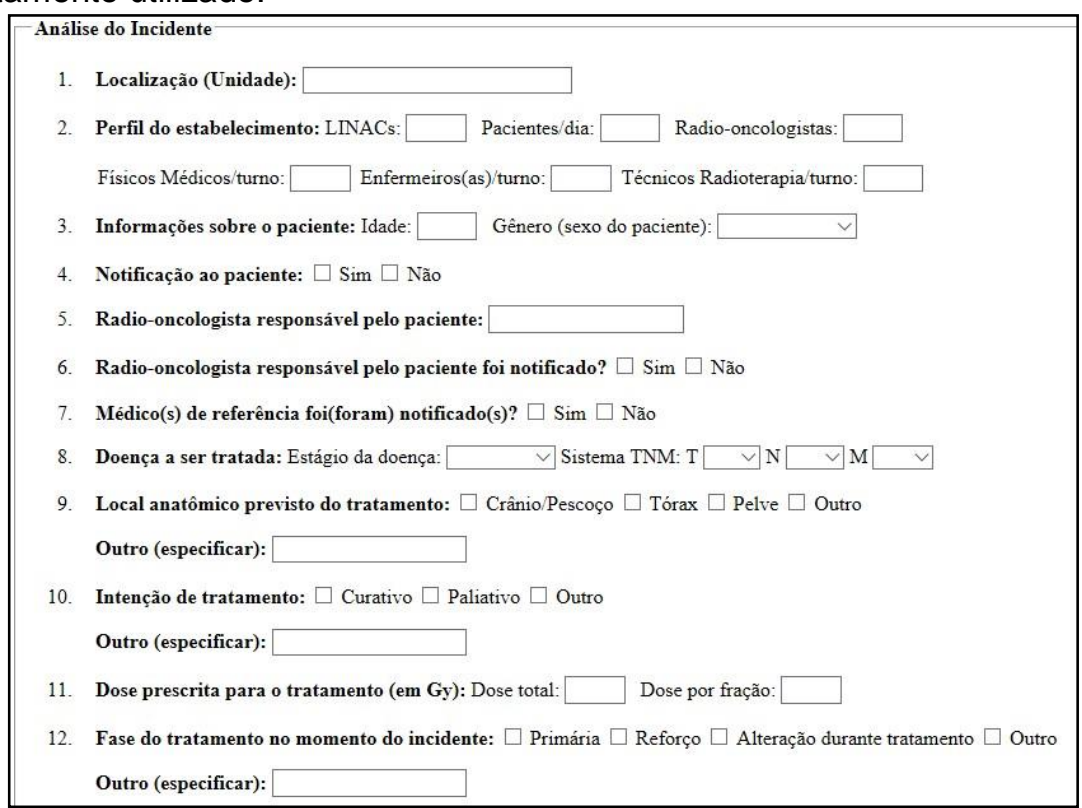

Figura 3: Formulário equivalente a análise de incidentes do SPIRad com campos referentes a técnicas de tratamento e detalhamento de equipamentos.

13. Técnica de tratamento utilizada ou planejada no momento do incidente:

$\square$ Simples $\square$ Conformacional 3D $\square$ IMRT $\square$ SRT/SRS Cranial $\square$ SBRT $\square$ VMAT

$\square$ Intracraniana, intraluminal, intravascular ou de superficie $\square$ LDR, PDR $\square$ HDR

$\square$ Implante temporário $\square$ Implante permanente $\square$ Ortovoltagem $\square$ Outro

Outro (especificar):

14. Tipo de imagem usada para localização da área a ser tratada:

$\square$ Radiografias de kV ou MV $\square$ Cone-beam CT $\square$ IGRT: MV cone-beam CT $\square$ MV CT $\square$ Outra Outra (especificar):

15. Função da pessoa que inicialmente descobriu o incidente:

$\square$ Radio-oncologista $\square$ Físico Médico $\square$ Enfermeiro(a) $\square$ Técnico Radioterapia $\square$ Outro Outro (especificar):

16. Unidade de tratamento:

Fabricante: $\square$ Modelo

17. Sistema de planejamento de tratamento:

Fabricante: $\square$ Modelo:

18. Sistema de registro e verificação:

Fabricante: $\square$ Modelo: 
Figura 4: Formulário equivalente a análise de incidentes do SPIRad com campos referentes às métricas de gravidade [DONALDSON, 2007; FORD et al., 2012].

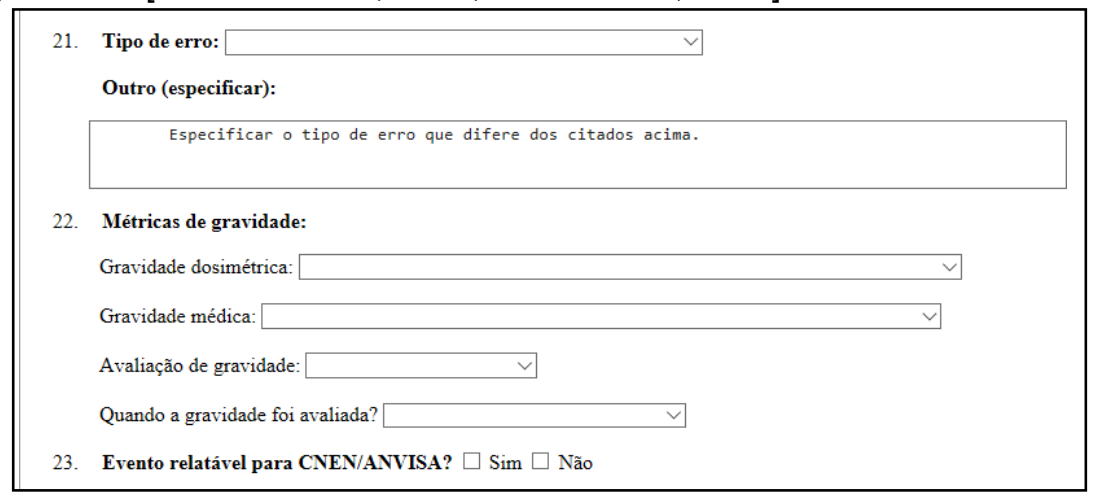

Figura 5: Formulário equivalente a análise de incidentes do SPIRad referentes aos campos dissertativos sobre o incidente.

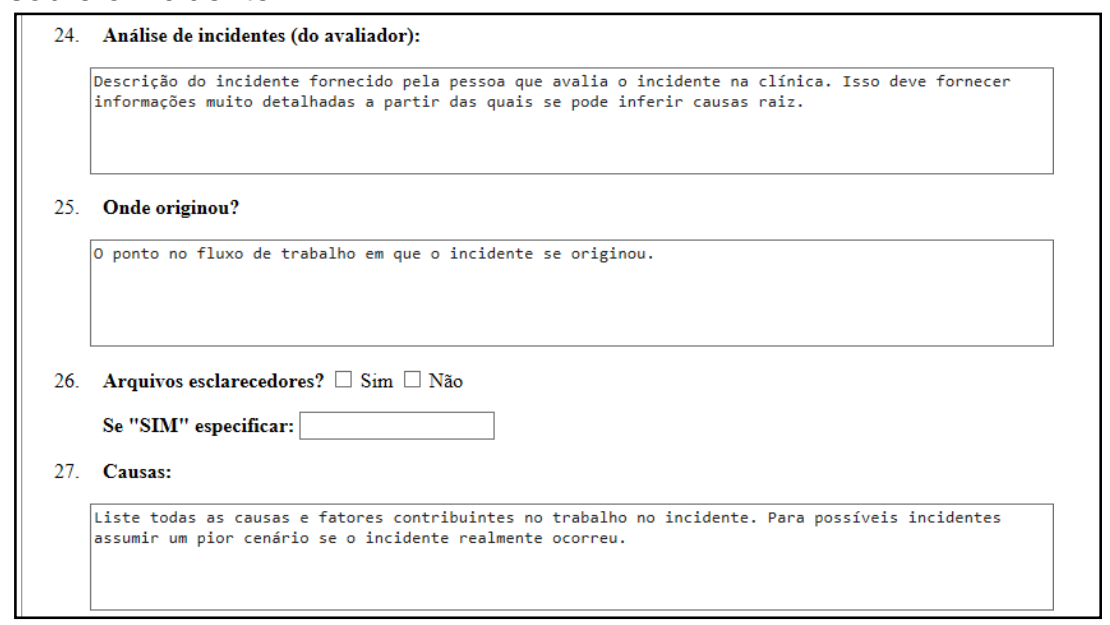

Figura 6: Formulário equivalente ao formulário de resposta do SPIRad.

\begin{tabular}{|l|l|} 
1. & Escala de ação clinica: $\square \mathrm{A} \square \mathrm{B} \square \mathrm{C} \square \mathrm{D}$ \\
2. & Intervenção: \\
& Outro (especificar): \\
3. & Barreiras de segurança: \\
& $\begin{array}{l}\text { As barreiras de segurança ou medidas de controle de qualidade no local que impediram ou poderiam } \\
\text { evitar o incidente. }\end{array}$ \\
4. Ação corretiva: \\
A(s) ação(ões) corretiva(s) tomadas para mitigar o dano para este paciente em particular. \\
\end{tabular}


Figura 7: Últimos campos do formulário de resposta do SPIRad.

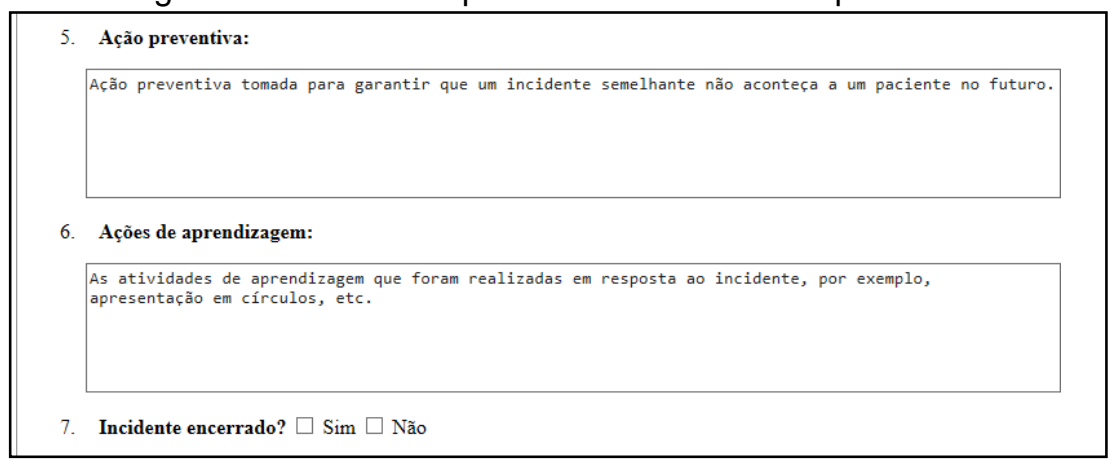

No formulário referente ao resumo do incidente, ao selecionar no campo "Tipo de Incidente" a opção "near-miss (um quase acidente)", os 3 formulários se resumem a apenas 1 formulário com a inserção de campos dissertativos específicos à opção selecionada, conforme Figuras 8 e 9.

Figura 8: Campos dissertativos do formulário para near-misses do SPIRad.

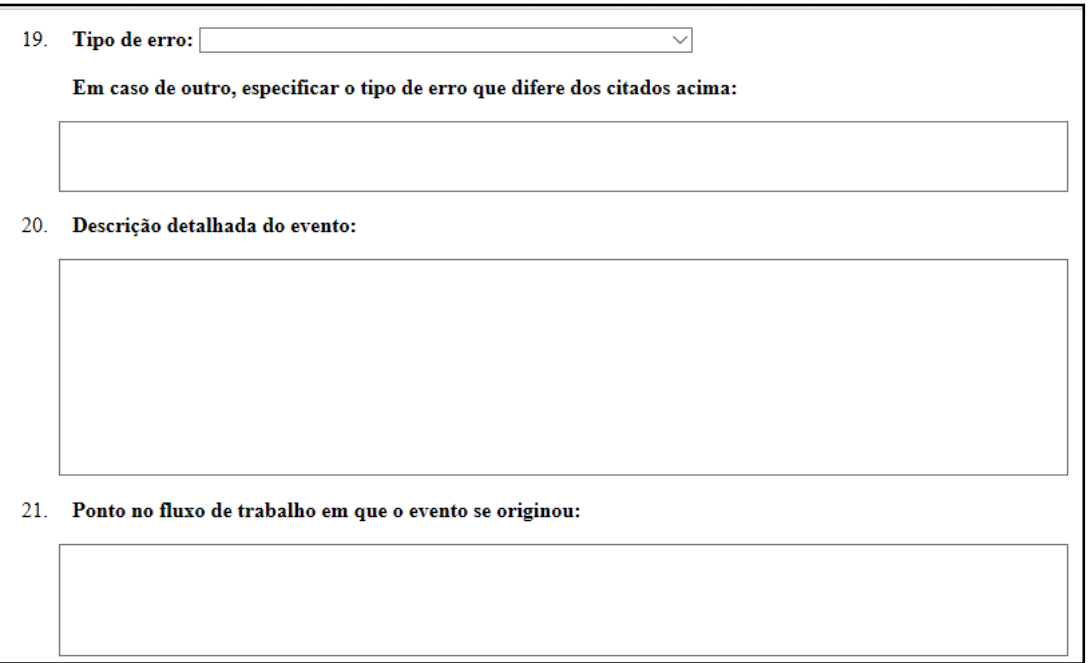


Figura 9: Últimos campos do formulário para near-misses do SPIRad.

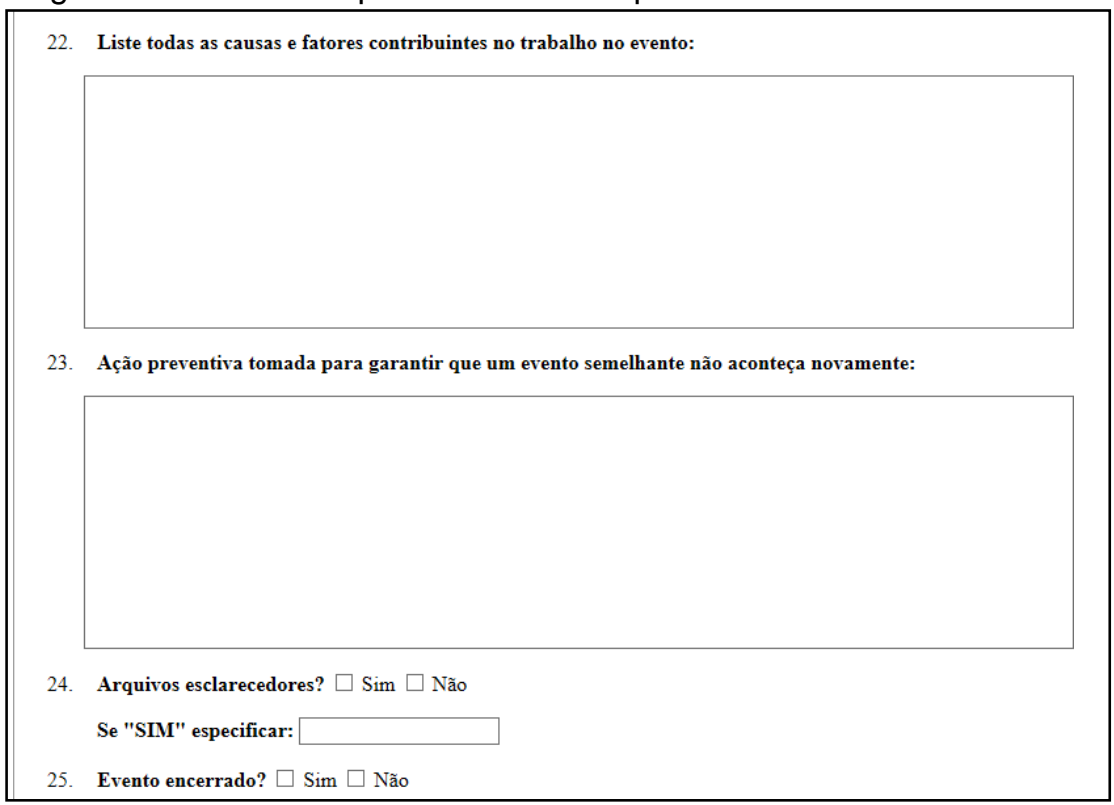

Para as escalas métricas de gravidade, foi adotada a terminologia do American Consensus em conjunto com o Toward Safer Radiotherapy [DONALDSON, 2007; FORD et al., 2012] que destaca como referência o termo "incidente", que pode ser um erro ou um evento adverso, como algo passível de interesse para a metodologia, mesmo que as consequências clínicas sejam menores [FORD et al., 2012]. Na publicação é possível encontrar a definição para alguns termos já vistos em publicações referentes a essa temática e de comum dificuldade de interpretação. As escalas métricas de gravidade adaptadas desta publicação para o trabalho foram separadas em escalas de gravidade dosimétrica variando dede nível 0 para "Não aplicável" até 9/10 para "100\% de desvio de dose em relação ao prescrito para qualquer estrutura", e gravidade médica variando desde nível 0 para "Nenhum dano causado" até nível 10 para "Morte prematura" [FORD et al., 2012].

\section{RESULTADOS}

As duas instalações que contribuíram para este trabalho foram denominadas como Instituição I e II (uma pública e a outra privada). A Instituição I atende uma demanda de 300 pacientes por dia distribuídos em 3 turnos, e sua equipe de radioterapia é composta por 39 profissionais, além de operarem com 5 aceleradores lineares e possuírem 1 HDR (high-dose rate brachytherapy). A 
Instituição II atende uma demanda de 75 pacientes por dia distribuídos em 4 turnos, e sua equipe de radioterapia é composta de 9 profissionais, operam com 1 acelerador linear e não possuem HDR.

Em ambas as instituições, a aplicação teste do SPIRad durou aproximadamente 5 meses e, durante esse período, tanto na Instituição I quanto na II os registros foram realizados por físicos médicos responsáveis pelas equipes que se dispuseram a colaborar com a pesquisa. Após a aplicação teste, foi possível detectar algumas características das instituições de acordo com os fatores críticos de sucesso para a implementação de um sistema de aprendizagem com incidentes. A análise permitiu fazer as seguintes afirmações para cada fator crítico:

Necessidade de recursos: devido à grande demanda de serviço nas duas instituições; Hábito de registro: os colaboradores tiveram dificuldade em interromper a rotina para poder alimentar o sistema com registros; Treinamento dos usuários e teste piloto: após apresentar o sistema e orientar sobre os campos e preenchimento em ambas as Instituições, os colaboradores utilizaram o SPIRad para "migrar" do livro de ocorrências para o sistema alguns registros realizados anteriormente; Facilidade de registro, definições e escopo: a partir do teste piloto, foi possível mediante feedbacks realizar updates necessários em alguns campos que, até o momento, estavam gerando dúvidas por parte de alguns integrantes de ambas as equipes colaboradoras; Inclusão de near-misses: a maior parte dos registros coletados para análise foram referentes a near-misses; Registros voluntários e não punitivo: pelo fato de ter sido uma aplicação teste, os colaboradores poderiam registrar anonimamente e não haveria identificação em caso de não preenchimento de incidentes reais e near-misses; Anonimização dos dados: ao analisar as causas dos eventos, o fator "erros humanos" foi predominante em quase todos os registros coletados, tantos incidentes reais quanto near-misses; Foco na melhoria do sistema e qualidade do registro: os colaboradores de ambas as Instituições enfatizaram que o objetivo do sistema é a melhoria da qualidade e da segurança nos processos; Percepção do erro: todos os registros foram realizados por físicos médicos de ambas as equipes das Instituições I e II, porém em boa parte dos registros os técnicos de radioterapia foram responsáveis por detectar o incidente, o que mostra a percepção do erro 
por parte de outros integrantes da equipe; Apoio organizacional: Na Instituição II, após reunião com físicos e superiores, obteve-se o apoio da alta direção para que pudesse ser possível utilizar as instalações para realizar a aplicação teste do SPIRad; entretanto, na Instituição I ocorreu apenas uma reunião com o chefe do setor de física médica para que fosse possível utilizar a instalação; Integração com o sistema de registro geral do hospital/Instituição: os colaboradores da Instituição II aproveitaram a aplicação teste para oficializar os registros relatados e imprimiram os eventos registrados e anexaram ao livro de ocorrências e surgiu a proposta de implementar o banco de dados do SPIRad na Instituição e em suas filiais.

Ao avaliar o tópico referente às métricas de gravidade nos registros coletados, foram coletadas as seguintes informações referentes aos incidentes registrados:

Todos os incidentes reais tiveram escala de gravidade dosimétrica de 1/2 (desvio absoluto de dose da prescrição total abaixo de 5\%);

- Todos os incidentes reais tiveram escala de gravidade médica de 0 (sem danos);

- Todos os incidentes reais tiveram a gravidade avaliada em menos de $24 \mathrm{~h}$ após o incidente;

- A escala de ação clínica em todos os registros de incidentes reais foi de "A" na relação de $A$ até $D$, ou seja, tiveram uma escala baixa.

Os incidentes registrados no SPIRad durante toda a aplicação foram aqueles que tiveram impacto, ou potencial de impactar a saúde física de pacientes assim como danos a equipamentos devido à má utilização ou descuido que pudessem, de alguma forma, impactar a saúde física dos pacientes. Na maioria dos sistemas descritos na literatura, o grupo de profissionais que mais geram registros de incidentes são técnicos de radioterapia, dosimetristas e físicos médicos, sendo que a participação de médicos é quase inexistente. Entretanto, conforme descrito anteriormente nos fatores críticos detectados na aplicação do SPIRad, devido aos motivos citados, poucos resultados foram coletados, totalizando 10 registros (4 da Instituição I e 6 da Instituição II), gerando uma baixa estatística.

Todos os registros obtidos nas Instituições I e II foram preenchidos por 
físicos médicos, pois os mesmos optaram por ficarem responsáveis por essa função de alimentar o SPIRad com informações. Ao fazer o levantamento dos registros, observou-se que:

- $70 \%$ dos incidentes registrados foram detectados por técnicos de radioterapia na sala de comando durante o tratamento, sendo que $35 \%$ foram identificados como incidentes reais e 35\% como near-misses;

- $30 \%$ dos incidentes registrados foram detectados por físicos médicos no planejamento do tratamento, sendo todos near-misses;

- Todos os incidentes registrados foram referentes a tratamento por feixe externo, durante a aplicação teste não foi registrado nenhum incidente referente a braquiterapia.

Existem vários fatores que podem contribuir para um incidente e um nearmiss. Em atenção aos campos referentes às causas e fatores que contribuíram para a ocorrência do incidente nas duas Instituições, verifica-se que:

- $60 \%$ dos fatores contribuintes encontrados nos registros estão relacionados a erros humanos, o que equivale a 6 registros dos 10 analisados;

- $\quad 30 \%$ dos fatores estão relacionados a falhas em procedimentos, 0 que equivale a 3 registros dos 10 analisados;

- Apenas $10 \%$ dos fatores estão relacionados a problemas técnicos em equipamentos, o que equivale a apenas 1 registro dos 10 analisados.

Ao fazer o levantamento dos tipos de erros, foi feita uma análise dos registros coletados. Dentre os tipos de erros relatados, os mais encontrados foram:

Paciente errado: 2 incidentes resultando em incidentes reais que foram sinalizados por técnicos de radioterapia durante o processo; Prescrição de dose: 2 incidentes apresentaram esse tipo de erro na etapa de revisão e verificação para pré tratamento, ambos sinalizados por físicos médicos e caracterizados como near-misses; Erro de delineamento: 1 incidente apresentou esse tipo de erro na etapa de revisão e verificação para pré tratamento, foi sinalizado por um físico médico e classificado como um near-miss; Abertura de plano de tratamento errado/antigo de paciente: 2 incidentes apresentaram esse tipo de erro na etapa de entrega do tratamento, foram sinalizados por técnicos de radioterapia e resultaram em 1 incidente real e 1 near-miss; Erro de conferência dos planos de 
tratamento do paciente: 3 incidentes apresentaram esse tipo de erro na etapa de entrega do tratamento, foram sinalizados por técnicos de radioterapia e foram classificados como 1 incidente real e 2 near-misses.

As barreiras de segurança são importantes pontos críticos de controle no mapa de processo do tratamento de radioterapia e possuem, como principal função, evitar que erros ocorram durante o processo e é fundamental que seja percebido de até que ponto estas são ou não eficazes para que seja possível analisar a informação. As barreiras de segurança estabelecidas nos registros de ambas as Instituições foram analisadas relativamente à capacidade de sucesso/falha na detecção de incidentes e near-misses. É possível verificar que existem barreiras bastante eficientes na detecção de incidentes; entretanto, é importante destacar que as barreiras que não detectaram um incidente não são necessariamente falhas, elas apenas não contribuíram para a detecção por não se relacionarem diretamente com o incidente, podendo ser consideradas barreiras fracas para o incidente relatado.

Ao analisar as barreiras relatadas nos registros coletados, detectou-se que as barreiras de segurança "Monitorização do tratamento" e "Verificação dos dados na folha de tratamento" foram as mais eficientes na detecção dos incidentes registrados sendo um incidente real ou near-miss. A barreira denominada "Verificação de ID do Paciente", foi uma barreira de segurança redundante, estando presente em praticamente todas as etapas do mapa de processo de tratamento. As duas últimas barreiras não detectaram erros, isso provavelmente se deve ao fato de estas não terem sido necessárias já que as barreiras que antecedem as mesmas foram eficazes na detecção do incidente.

Por fim, ao levantar os dados referentes aos campos do formulário que focam em ações corretivas, preventivas e de aprendizagem, todos os 10 registros apresentaram soluções referentes aos incidentes registrados. Os incidentes que apresentaram como erro "paciente errado" buscaram como ação corretiva o reforço das ações de identificação do paciente e melhoria na organização da agenda de tratamento, tendo como ação preventiva o aprimoramento na exigência de foto do paciente no sistema de gerenciamento, e como ação de aprendizagem a melhoria da comunicação de defesa em profundidade. Os incidentes que apresentaram como erro "prescrição errada" e "erro de delineamento" tiveram 
apenas como ação preventiva respectivamente o aprimoramento do uso de prescrição eletrônica pelos médicos no sistema de gerenciamento e a utilização de conjuntos de estrutura para delineamento padronizado e implantação de dupla verificação médica. Os incidentes que apresentaram como erro "abertura de plano errado/antigo do paciente" foram aqueles que tiveram como fatores problemas técnicos e tiveram como ação preventiva a inserção de forma manual do tratamento no sistema de gerenciamento e finalização de todos os cursos de tratamento após o término do plano de tratamento de modo a não o deixar mais disponível para ser aberto erroneamente na sala de comando. Por fim, os incidentes que apresentaram como erro "conferência dos planos de tratamento" tiveram como ação corretiva o agendamento de uma nova aplicação ao final do curso de tratamento do paciente para finalizar de forma completa o tratamento e como ação preventiva a necessidade da presença do físico médico também nas mudanças de fase do tratamento.

\section{CONCLUSÕES}

Por meio de um estudo realizado, de uma revisão e de uma harmonização de todos os conceitos, foi possível estabelecer critérios de estruturação para uma plataforma de registro de incidentes nas Instituições colaboradoras. O sistema de aprendizagem com incidentes é uma importante ferramenta utilizada na gestão de risco e conforme descrito na literatura a aplicação desta metodologia tem sido recomendada por especialistas na área da saúde e destaca-se na radioterapia, conforme exigido por instituições reguladoras. Após a conclusão do ambiente digital do SPIRad e de sua aplicação teste, é possível afirmar que o SPIRad poderá ser de grande utilidade no aprimoramento de protocolos de tratamento, uma vez que será possível além de reportar, também analisar os eventos para obtenção de informações que impactam no tratamento, possibilitando empregar medidas para diminuir os riscos. O SPIRad permite um registro sequencial de incidentes e near-misses em radioterapia, dividido em categorias e processos ao longo do tratamento. Mediante feedbacks dos usuários, foi possível observar que a praticidade da ferramenta foi uma das características mais mencionadas e tal qualidade é comprovada nos registros, visto que não houve erros de preenchimento, mesmo a aplicação teste ter sido realizada de forma offline. Pelo 
fato de ser uma ferramenta voluntária e com característica instrutiva e não punitiva, o SPIRad pode auxiliar na política de gestão de qualidade e na cultura de segurança do paciente. 


\section{REFERÊNCIAS}

Begg, a. C.; stewart, f. A.; vens, c. Strategies to improve radiotherapy with targeted drugs. Nature review cancer v. 11, 239-253, 2011.

Delaney, g. Et al. The role of radiotherapy in cancer treatment: estimating optimal utilization from a review of evidence-based clinical guidelines. Cancer, v. 104, p. 1129-37, 2005.

Donaldson, s. Towards safer radiotherapy. British institute of radiology, institute of physics and engineering in medicine, national patient safety agency, society and college of radiographers, the royal college of radiologists, london, 2007.

Ford, e. C. Et al. Consensus recommendations for incident learning database structures in radiation oncology. Medical physics v. 39, p. 7272-7290, 2012.

Hendee, w. R.; herman, m. G. Improving patient safety in radiation oncology. Medical physics, v. 38, p. 78-82, 2011.

Holmberg, o.; mcclean, b. Preventing treatment errors in radiotherapy by identifying and evaluating near misses and actual incidents. Journal of radiotherapy in practice, v. 3, p. 13-25, 2006.

Icrp. Prevention of accidental exposures to patients undergoing radiation therapy. Annals of the icrp, v. 30, p. 7-7, 2000.

Cnen. Norma cnen nn-6.10. Requisitos de segurança e proteção radiológica para serviços de radioterapia, 2014. 